\title{
Photoluminescence of high optical quality CdSe thin films deposited by close-spaced vacuum sublimation
}

\author{
Yu.P. Gnatenko ${ }^{\text {a,* }}$, P.M. Bukivskij ${ }^{\text {a }}$, I.O. Faryna ${ }^{\text {a }}$, A.S. Opanasyuk ${ }^{\mathrm{b}}$, M.M. Ivashchenko ${ }^{\mathrm{b}}$ \\ a Institute of Physics of National Academy of Sciences of Ukraine, Kyiv 03028, Ukraine \\ ${ }^{\mathrm{b}}$ Sumy State University, Rymsky-Korsakov Street 2, Sumy 4007, Ukraine
}

\section{A R T I C L E I N F O}

\section{Article history:}

Received 7 June 2013

Accepted 25 September 2013

Available online 8 October 2013

Keywords:

II-VI films

Low-temperature photoluminescence

Residual impurity states

Point defects

Optical quality

\begin{abstract}
A B S T R A C T
Polycrystalline CdSe thin films ( $d=0.1-3.0 \mu \mathrm{m}$ ) have been deposited on a glass substrate by means of the close-spaced vacuum sublimation technique. X-ray diffraction measurements have shown that the films obtained at $T_{s}>473 \mathrm{~K}$ have only wurtzite phase. The influence of deposition conditions, in particular, the substrate temperature on the photoluminescence (PL) of CdSe films spectra was investigated. This let us study the effect of glass substrate on their optical quality as well as determine the nature and energy structure of the intrinsic defects and residual impurities in the films. The presence in PL spectrum of the most intense sharp donor bound exciton $\mathrm{D}^{0} \mathrm{X}$-line for CdSe films obtained at $T_{s}=873 \mathrm{~K}$ indicates the n-type conductivity and their high optical quality. Intensive PL bands in the spectral range 1.65-1.74 eV were also observed, which are associated with the recombination of donor-acceptor pairs with the participation of the shallow donor and acceptor centers caused by $\mathrm{Na}(\mathrm{Li})$ residual impurities. As a result of the study of the PL spectra of CdSe films the optimal temperature conditions of their growth were determined, namely, the substrate temperature $T_{s}=873 \mathrm{~K}$ and the evaporator temperature $T_{e}=973 \mathrm{~K}$.
\end{abstract}

(c) 2013 Elsevier B.V. All rights reserved.

\section{Introduction}

CdSe films with direct band gap energy of about $1.75 \mathrm{eV}$ at room temperature have n-type conduction and high photosensitivity in the visible spectral region. At present, such films are intensively studied mainly because they are suitable material for elaboration of a number of optoelectronic devices such as photoelectrochemical solar cells, photo- and gas- detectors, highperformance thin film transistors, gamma ray detectors, lightemitting diodes, etc [1-3]. Furthermore, CdSe thin films can also be used as the absorber layers in the top of tandem solar cells and they are considered to be an important material for photovoltaic applications. That is caused by a high absorption coefficient of CdSe films and nearly optimize their band gap energy for coefficient conversion of light absorption to electrical power.

In order to effectively use those thin films in micro- and optoelectronics it is necessary to optimize their optical, structural and electronic properties. It should be noted that for optoelectronic applications it is very important to have a transparent substrate to the intrinsic (excitonic) light emitted by the active area of thin films. It was found that CdSe films deposited on silicon [4], titanium [5,6] and GaAs [7] substrates manifest intrinsic luminescence, whereas the films deposited on quartz or glass

\footnotetext{
* Corresponding author. Tel.: +38044525 4717.

E-mail address: yuriygnatenko@ukr.net (Yu.P. Gnatenko).
}

substrates do not show this luminescence [8]. Therefore, it is very important to obtain CdSe films of high optical quality by their deposition on the quartz or glass substrates which are transparent for CdSe intrinsic emission. Earlier [9-11], it was shown that a low cost close-spaced vacuum sublimation (CSVS) method makes it possible to deposit the stoichiometric films of binary II-YI semiconductors with the controllable physical properties using glass as substrate. However, until now there was very little study of CdSe polycrystalline films deposited using this method.

The important factor responsible for the physical properties of thin films is their crystal structure quality. The intrinsic defects in deposited CdSe thin films are randomly distributed on the surface and in the volume of the films. They have strong effect on the optical and photoelectrical properties of thin films and thus considerably affect on their application efficiency.

Low temperature photoluminescence $(\mathrm{PL})$ measurements is a very effective method for studying the defect structure of various semiconductor materials including polycrystalline thin films [9,12-14]. This makes it possible to determine the nature and energy levels of both the intrinsic defects and the residual impurities in the semiconductor materials as well as the relative concentration of dislocations in the films. Currently the PL properties of bulk crystals of II-YI group semiconductors are studied well enough [15-17], but at the same time thin films of these materials have not been thoroughly investigated.

In this work, we study the PL properties of CdSe films deposited on the glass substrates by means of the CSVS technique. This let us 
determine the nature and energy levels of point defects and the presence of dislocations in the films as well as study the effect of growth conditions on their defect structure. As a result of those studies the deposition conditions of CdSe thin films of high optical quality were established.

\section{Experimental details}

CdSe polycrystalline films were deposited by CSVS method $[9,11]$ on the cleaned glass substrates under different growth conditions, namely: the evaporator temperature was $T_{e}=973 \mathrm{~K}$, the substrate temperature $T_{s}$ varied from $473 \mathrm{~K}$ to $873 \mathrm{~K}$, the growth time was $10 \mathrm{~min}$. The stoichiometric powder of CdSe as an initial material was used. The CSVS method is described in detail in our work [9].

Surface morphology was investigated by scanning electron microscopy (SEM). Average grain size $(d)$ in the layers was estimated by the Jeffries method [18]. Thickness of the films $(l)$ was measured by the SEM directly from the samples cross-section. $\mathrm{X}$-ray method was applied for determination of the microstress level $\varepsilon$ in CdSe thin films. The PL spectra were measured using an SDL-1 grating spectrometer [12,13,19]. An LGN-404 argon laser was used for excitation by the $488.8 \mathrm{~nm}$ line, together with an FEU-62 photomultiplier in the photon-counting mode. The samples were mounted on the cold finger of a variable temperature liquid-helium cryostat. The accuracy of the measurements and temperature stabilization by the UTREKS system was $0.01 \mathrm{~K}$. The spectral resolution of the system was of the order of $0.1-0.2 \mathrm{meV}$.

\section{Results and discussion}

\subsection{Characterization of CdSe thin films}

It has been found that the films obtained at low substrate temperatures $\left(T_{s}<673 \mathrm{~K}\right)$ have a highly dispersed structure with the grain size $(d)$ from $0.1 \mu \mathrm{m}$ to $3.0 \mu \mathrm{m}$. As can be seen from Fig. 1, where the surface morphology of the investigated CdSe films is shown, for the film deposited at $T_{s}=673 \mathrm{~K}$ the layer-by-layer mechanism determines the layers growth process (c).

When we increase the substrate temperature, the growth mechanism changes. At $T_{s}=873 \mathrm{~K}$ (b) the films have a columnarlike structure (d) with the clear growth texture. For $T_{e}=973 \mathrm{~K}$ and $T_{s}=873 \mathrm{~K}$ CdSe films with grain size $d=(3-4 \mu \mathrm{m})$ and layers thickness $l=(5-6 \mu \mathrm{m})$ were obtained. X-ray diffraction of CdSe films have shown that they correspond to only single wurtzite phase. It was also found that the CdSe thin films deposited at $T_{s}<473 \mathrm{~K}$ are bi-phase. At the same time, for high temperature regime of film growth $\left(T_{s}>473 \mathrm{~K}\right)$ there is only wurtzite phase. It was shown that the microstress level $\varepsilon$ in CdSe thin films decreases with increasing the substrate temperature. In particular, for CdSe thin films deposited at $T_{s}$ equal to $773 \mathrm{~K}$ and $873 \mathrm{~K}$ these values correspond to $4.0 \times 10^{-3}$ and $0.5 \times 10^{-3}$, respectively.

\subsection{Photoluminescence of CdSe films}

Fig. 2 shows PL spectra of the CdSe polycrystalline films at $4.5 \mathrm{~K}$ for the samples obtained at different $T_{S}$ (curves 1 and 2 correspond to $893 \mathrm{~K}$ and $773 \mathrm{~K}$, respectively). As can be seen from Fig. 2 (curve 1), relatively broad group of PL bands in the spectral range 1.65-1.75 eV are observed. The highest intensity $\mathrm{I}_{\mathrm{DAP} 1}$-line at $1.7357 \mathrm{eV}$ is observed, which can be caused by the free-to-band or band-to-band optical transitions. It should be noted that here other lines at $1.7095 \mathrm{eV}$, $1.6833 \mathrm{eV}$ and $1.6571 \mathrm{eV}$ show up which correspond to 1LO-, 2LO- and 3LO- phonon replicas of the IDAP1-line.

The presence of these lines is characteristic of the bound-tobound transitions with the participation of donor-acceptors pairs (DAPs) $[4,6,20]$. In this case the energy of the emitted light, which is associated with the zero-phonon I IAP1-line, is given by the following equation $[21,22]$ :

$E=E_{g}-\left(E_{D}+E_{A}\right)+e^{2} / \varepsilon_{0} \varepsilon R$

where $E_{g}$ is the band gap energy, $E_{D}$ and $E_{A}$ are the ionization energies of donor and acceptor centers, respectively, $e^{2} / \varepsilon_{0} \varepsilon R$ is the
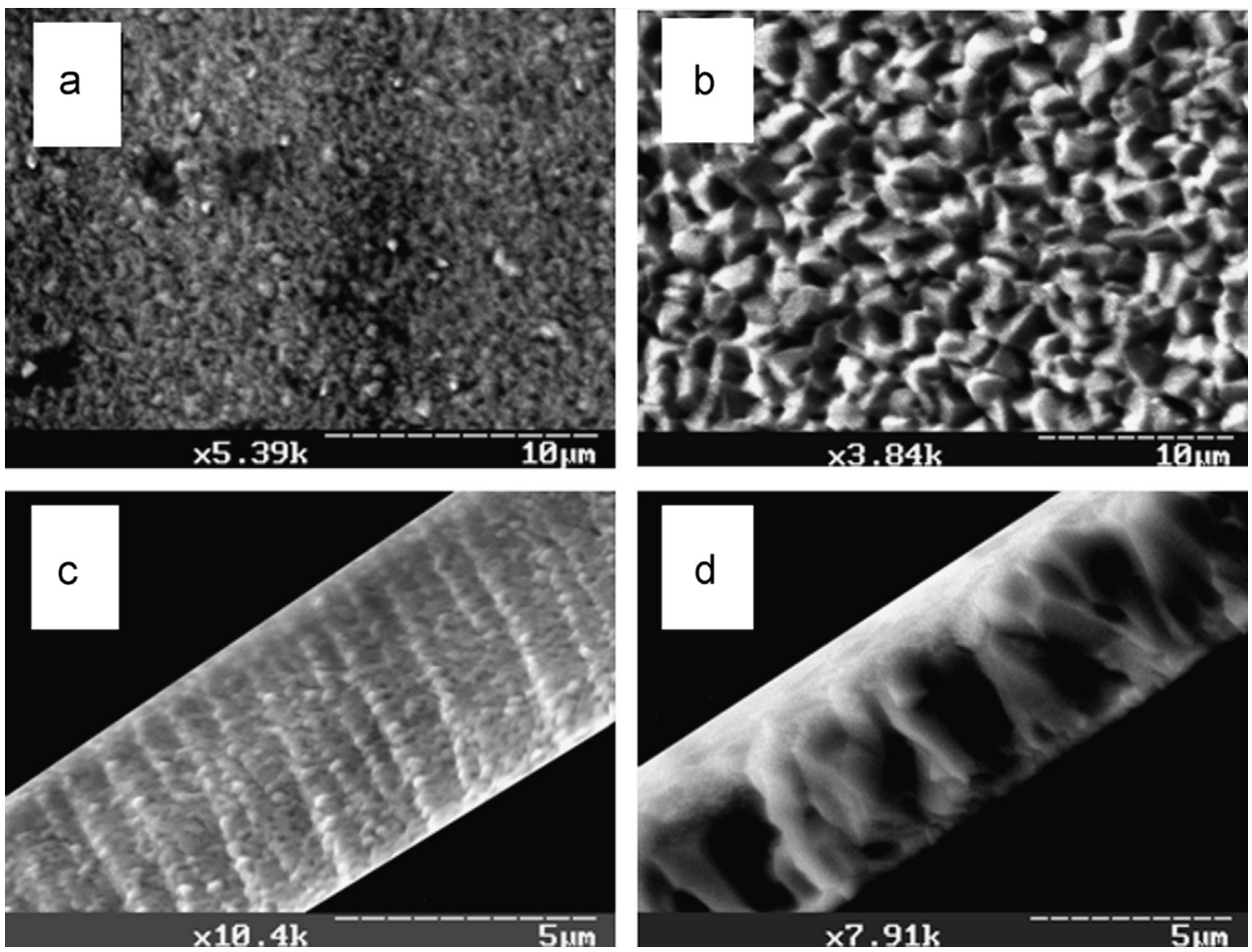

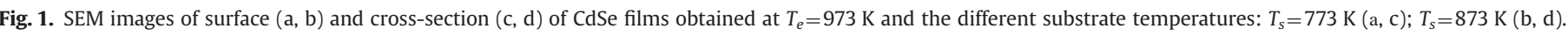




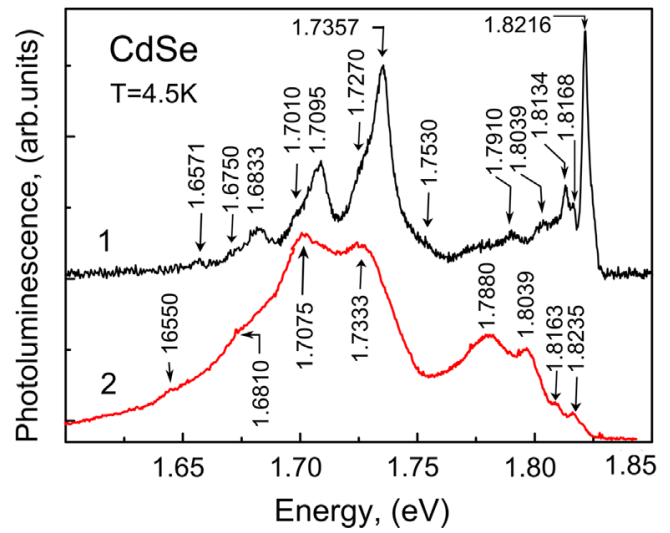

Fig. 2. Low-temperature $(T=4.5 \mathrm{~K}) \mathrm{PL}$ spectra for CdSe polycrystalline films grown by the CSVS technique under different technological conditions: $T_{e}=973 \mathrm{~K}$; curves $1-2$ for $T_{s}=873 \mathrm{~K}$ and $773 \mathrm{~K}$, respectively.

Coulomb energy of the two ionized impurity centers (donor and acceptor pairs) separated by distance $R, \varepsilon_{0} \varepsilon$ is the dielectric constant of the investigated material, $e$ is the elementary charge. The ionization energy of shallow acceptors in CdSe is $109 \mathrm{meV}$ [23]. This acceptor center is associated with Li or Na substituted into the cation sites, i.e. $\mathrm{Li}_{\mathrm{Cd}}$ or $\mathrm{Na}_{\mathrm{Cd}}$ centers. These impurity atoms are the typical residual impurities in II-YI compounds [24]. The ionization energy of shallow donor centers, which can be caused with the interstitial $\mathrm{Li}$ atoms $\left(\mathrm{Li}_{\mathrm{i}}\right)$, is $19.0 \mathrm{meV}$ [25]. Using in $\mathrm{Eq}$. (1) the values of $109 \mathrm{meV}$ and $19.0 \mathrm{meV}$ for the acceptor and donor binding energies, respectively, $1.841 \mathrm{eV}$ [26] for the band gap energy and 10 [27] for the dielectric constant the value $R_{1}=6.3 \mathrm{~nm}$ was obtained.

As can be seen from Fig. 2, the bends at 1.727, 1.701 and $1.675 \mathrm{eV}$ are observed on the long-wavelength wing of the IDAP1line and its LO-phonon replicas. They can be also caused by the DAP2 recombination which arises from another set of donor or acceptor levels with the energies even closer to the conduction or the valence bands. It should be noted that the presence of $P$ atoms in CdSe induces the appearance of the shallow acceptor level with the energy $E_{\mathrm{V}}+0.083 \mathrm{eV}$ [28]. Thus, it may be possible that the DAP2 recombination with the participation of this acceptor center occurs. Usually, in this case the band at $1.753 \mathrm{eV}$ appears in $\mathrm{PL}$ spectrum of CdSe containing P atoms [23]. As can be seen from Fig. 2, the bend at $1.753 \mathrm{eV}$ is observed in the investigated spectrum which may be identified with the $\mathrm{I}_{\mathrm{DAP2}}$-zero-phonon line. Other lines at $1.727 \mathrm{eV}, 1.701$, and $1.675 \mathrm{eV}$ correspond to $1 \mathrm{LO}-$ , 2LO-, and 3LO- phonon replicas of this line. The observation of this emission could indicate the presence of a small concentration of the residual $\mathrm{P}$ atoms in $\mathrm{CdSe}$ film.

Usually, CdSe has excess Se vacancies over Cd vacancies and thus exhibits deviation from stoichiometry. Therefore, it is expected that excess $\mathrm{Cd}$ in the CdSe film produces Se vacancies and/or $\mathrm{Cd}$ interstitial $\left(\mathrm{Cd}_{\mathrm{i}}\right)$, which induce the appearance of donors. The donor level for the DAP2 recombination may correspond to the interstitial $\mathrm{Cd}_{\mathrm{i}}$ atoms with the energy of $E_{\mathrm{c}}-0.014 \mathrm{eV}$ [28]. According to Eq. (1), taking into account the energies of the acceptor center caused by the presence of $\mathrm{P}$ atom $\left(E_{\mathrm{V}}+0.083 \mathrm{eV}\right)$ and the energy of the donor centers $\left(E_{\mathrm{c}}-0.014 \mathrm{eV}\right)$, we get for DAP2 the values of $R_{2}$ to be $15.2 \mathrm{~nm}$.

In addition to the shallow donor and acceptor levels CdSe films can also contain deeper levels. Usually, the presence of these states leads to the appearance of PL bands located in the longwavelength spectral region at $1.50 \mathrm{eV}$ and $1.33 \mathrm{eV}$, which are associated with the $\mathrm{Ag}$ and $\mathrm{Cu}$ residual atoms, respectively [20]. As can be seen from Fig. 2 the PL bands in this spectral region don't appear. This indicates the absence of these impurities atoms in the investigated CdSe films. Other deep donor levels are due to the presence of Se vacancies with the energies $E_{\mathrm{c}}-0.22 \mathrm{eV}$ and $E_{\mathrm{c}}-0.40 \mathrm{eV}$ [29]. There are also deep donor levels with the following energies: $E_{\mathrm{c}}-0.12 \mathrm{eV}$ and $E_{\mathrm{c}}-0.28 \mathrm{eV}$, which are the result of the structural defects introduced during the growth process [30]. As can be seen from the PL spectrum, presented in Fig. 2, these intrinsic defects are also absent in the investigated CdSe films.

In addition to PL bands caused by the participation of point intrinsic defects and residual impurities in the recombination process, the emission associated with extended defects like dislocations may arise in semiconductor polycrystalline films $[9,10]$. The appearance of dislocations in CdSe films can be caused by the appearance of microstress during the film growth. The transformation of the low-temperature PL spectra of CdSe crystals during their plastic deformations was observed in [31]. In particular, the emergence of the PL bands at $1.765 \mathrm{eV}$ and $1.792 \mathrm{eV}$ is caused by the presence of the motion of dislocations in the prismatic slip and the dislocations slip in the basic plane, respectively [31]. As can be seen from Fig. 2 (curve 1), PL spectrum of CdSe films deposited at $T_{\mathrm{s}}=873 \mathrm{~K}$ shows only the structure of weak intensity in the spectral region $1.75-1.81 \mathrm{eV}$. It is caused by the fact that the dislocation density in CdSe films deposited at this temperature is relatively small. The obtained result correlates with the microstress measurements of the investigated films, since the microstress level for the films grown at $T_{s}=873 \mathrm{~K}$ is minimal in comparison with the films obtained at lower substrate temperature.

More information on the presence and nature of intrinsic defects and residual impurities can be obtained from the analysis of the PL spectra in the exciton region. As can be seen from Fig. 2 (curve 1), the sharp line of high intensity is observed in the shortwavelength region of PL spectrum at $1.8216 \mathrm{eV}$. The energy position of this line indicates the appearance of the recombination of donor bound excitons, i.e. the presence of the $\mathrm{D}^{0} \mathrm{X}$-line which is characteristic for CdSe single crystals of n-type [21]. In this case the binding energy of exciton equals to $3.4 \mathrm{meV}$ because the energy of free exciton in CdSe single crystals is $1.825 \mathrm{eV}$ [32]. Earlier, this PL line is also observed in the CdSe films deposited by the laser ablation technique on a sapphire substrate [32]. In this case the intensity of $\mathrm{D}^{0} \mathrm{X}$-line was very small in comparison with the intensity of emission located at $1.75 \mathrm{eV}$ caused by DAP recombination involving shallow acceptor levels. It should be noted that its full width at half maximum (FWHM) was about $10 \mathrm{meV}$ [32]. In our case the FWHM of the $\mathrm{D}^{0} \mathrm{X}$-line is three times smaller and corresponds to about $3.0 \mathrm{meV}$. The fact that we are able to observe the sharp intensive excitonic line in the PL spectrum indicates that the CdSe films are of high optical quality. Furthermore, the presence of the $\mathrm{D}^{0} \mathrm{X}$-line shows that the films, obtained at $T_{s}=873 \mathrm{~K}$, are of n-type. Thus, it leads us to expect that the concentration of the neutral acceptor centers in the CdSe films is insignificant. The appearance of the $\mathrm{D}^{0} \mathrm{X}$-line in the PL spectrum of the film may be the result of the presence of the donor levels caused by the $\mathrm{Cd}_{\mathrm{i}}$ centers or $\mathrm{Na}(\mathrm{Li})$ interstitial $(\mathrm{Na}(\mathrm{Li}))_{\mathrm{i}}$ residual impurity. It should be noted that these donor centers were observed in CdSe crystals [20]. Other narrow lines of small intensity close to $\mathrm{D}^{0} \mathrm{X}$-line at $1.8168 \mathrm{eV}$ and $1.8134 \mathrm{eV}$ are also observed. They can be associated with the radiative recombination of an excitonic emission bound to shallow acceptors ( $\mathrm{I}_{1}$-line) caused by the presence of $\mathrm{Li}, \mathrm{Na}$ or $\mathrm{Cu}$ atoms. First line is in a good agreement with the energy of $\mathrm{I}_{1}$-line which appear in CdSe single crystals lighty doped by Li or Na atoms [20]. The presence of $\mathrm{P}$ atoms in CdSe induces the appearance in PL spectrum of $\mathrm{I}_{1}$-line at $1.8191 \mathrm{eV}$ [20]. As can be seen from Fig. 2 this line is not practically observed in PL spectrum of CdSe films deposited at $T_{s}=873 \mathrm{~K}$. 
In the case of the films obtained at $T_{s}=773 \mathrm{~K}$ (Fig. 2, curve 2) the intensity of $\mathrm{D}^{0} \mathrm{X}$-line at $1.8235 \mathrm{eV}$ is insignificant. This indicates that the donor concentration for CdSe films deposited at $T_{s}=773 \mathrm{~K}$ is considerably smaller than for the film obtained at $T_{s}=873 \mathrm{~K}$. It should be noted that in this case the intensity of $\mathrm{A}^{0} \mathrm{X}$-line at $1.8163 \mathrm{eV}$ is comparable with that for $\mathrm{D}^{0} \mathrm{X}$-line. According to Ref. [33] decrease of the donor concentration may be caused by increase of the dislocations concentration for the film obtained at $T_{s}=773 \mathrm{~K}$. At the same time, increase of the intensity of PL lines at $1.8039 \mathrm{eV}$ and $1.7880 \mathrm{eV}$, which can be associated with unknown acceptors, is observed. In this case, exciton-binding energies correspond to $21.1 \mathrm{meV}$ and $37.0 \mathrm{meV}$, respectively. Taking into account that there is correlation between the acceptor ionization energy $E_{A}$ and the exciton binding energy $E_{\mathrm{ex}}$, namely $E_{\mathrm{ex}} \approx 0.1 E_{A}$ [34] it is possible to estimate the value of $E_{A}$. In our case we found $E_{A}{ }^{1}=0.211 \mathrm{eV}$ and $E_{A}{ }^{2}=0.370 \mathrm{eV}$, respectively. The appearance of these acceptor levels may be caused with increase the dislocation density, since it was found that the microstress level increases from $0.1 \cdot 10^{-3}$ to $4.0 \cdot 10^{-3}$ for CdSe films deposited at $T_{s}=873 \mathrm{~K}$ and $T_{s}=773 \mathrm{~K}$, respectively. The high intensity PL bands at $1.7333 \mathrm{eV}$ and $1.7075 \mathrm{eV}$ are also observed which correspond to zero-phonon $\mathrm{I}_{\mathrm{DAP} 1}$-line and its 1LO-phonon replica. There are also 2LO-phonon and 3LO-phonon replicas located at $1.681 \mathrm{eV}$ and $1.655 \mathrm{eV}$, respectively. It is evident that the long-wavelength shift of the $\mathrm{I}_{\mathrm{DAP} 1}$-line is observed in comparison with the CdSe films obtained at $T_{s}=873 \mathrm{~K}$. It may be caused by increase the distance $R_{1}$ between donor-acceptor pairs of DAP1 type. In this case using Eq. (1) the value $R_{1}=7.0 \mathrm{~nm}$ was estimated. This indicates that the concentration of $\operatorname{Li}(\mathrm{Na})$ residual impurities for the films obtained at $T_{s}=773 \mathrm{~K}$ is smaller than that for deposited at $T_{s}=873 \mathrm{~K}$. This fact may be due to a higher diffusion of impurity atoms from the charge container at $T_{s}=873 \mathrm{~K}$.

In summary, we study the low-temperature PL properties of CdSe films deposited on transparent glass substrate at different temperatures by means of the close-spaced vacuum sublimation technique. Structural analysis showed that the films deposited at $T_{S}>473 \mathrm{~K}$ have only wurtzite phase. It was found that the PL spectrum of CdSe films deposited at $T_{S}=873 \mathrm{~K}$ exhibit sharp intense donor bound exciton $\mathrm{D}^{0} \mathrm{X}$-line, which is characteristic for CdSe single crystals of n-type, as well as other bands caused by donor-acceptor emission with the participation of $\mathrm{Na}(\mathrm{Li})$ resudial impurities. The presence of excitonic emission indicates the high optical quality of the investigated films. This results correlates with the strong decrease of the microstress level in CdSe thin films obtained at $T_{s}=873 \mathrm{~K}\left(0.5 \times 10^{-3}\right)$ in comparison with the films deposited at $T_{s}=773 \mathrm{~K}\left(4.0 \times 10^{-3}\right)$. Our findings provide a way to improve structural properties of polycrystalline CdSe films deposited on a glass substrate which is transparent for CdSe intrinsic emission to obtain high optical quality films suitable for optoelectronic and photovoltaic applications. In future, more detailed study of the structural properties of CdSe films obtained at different growth conditions will be carried out.

\section{Acknowledgments}

This research has been supported by the Ministry of Education and Science of Ukraine (Grant No. 0110U001151) by the National Academy of Sciences of Ukraine (Grants Nos. BC-157-15 and B-146-15).

\section{References}

[1] P. Mahawela, G. Sivaraman, S. Jeedigunta, J. Gaduputi, M. Ramalingam, S. Subramanian, S Vakkalanka, C.S. Ferekides, D.L. Morel, Materials Science and Engineering B 116 (2005) 283.

[2] S. Kasap, P. Capper, The Springer Handbook of Electronic and Photonic Materials, Springer, Berlin, 2007.

[3] M. Ohring, The Materials Science of Thin Films, Academic press, New York, 1992.

[4] G. Perna, V. Capozzi, M. Ambrico, Journal of Applied Physics 83 (1998) 3337.

[5] R. Jäger-Waldau, N. Stücheli, M. Braun, M. Lux Steiner, E. Bucher, Journal of Applied Physics 65 (1988) 2601.

[6] R.P. Siberstein, Micha Tomkiewicz, Journal of Applied Physics 54 (1983) 5428.

[7] M. Ambrico, V. Stagno, D. Smaldone, R. Martino, G. Perna, V. Capozzi, Proceedings of SPIE 3404 (1998) 39.

[8] G. Perna, S. Pagliara, V. Capozzi, M. Ambrico, T. Ligonzo, Thin Solid Films 349 (1999) 220.

[9] V. Kosyak, A. Opanasyuk, P.M. Bukivskij, Yu.P. Gnatenko, Journal of Crystal Growth 312 (2010) 1726.

[10] Yu.P. Gnatenko, P.M. Bukivskij, A.S. Opanasyuk, D.I. Kurbatov, M.M. Kolesnyk, V.V. Kosyak, H. Khlyap, Journal of Luminescence 132 (2012) 2885.

[11] D.I. Kurbatov, V.V. Kosyak, M.M. Kolesnyk, A.S. Opanasyuk, S.N. Danilchenko, Yu.P. Gnatenko, Materials Chemistry and Physics 138 (2013) 731.

[12] Yu.P. Gnatenko, P.M. Bukivskij, Yu.P. Piryatinski, I.O. Faryna, O.A. Shigiltchoff, R.V. Gamernyk, Applied Physics Letters 95 (2009) 112109.

[13] Yu.P Gnatenko, M.S. Brodyn, I.O. Faryna, P.M. Bukivskij, O.A. Shigiltchoff, M.S. Furyer, R.V. Gamernyk, N. Kukhtarev, T. Kukhtareva, Physica Status Solidi 204 (2007) 2431.

[14] M.S. Furyer, P.A. Skubenko, P.M. Bukivskij, L.M. Tarakhan, E.D. Chesnakov, I.G. Vertygel, A.I. Ovcharenko, L.S. Ivanova, R.V. Gamernyk, Yu.P Gnatenko, Journal of Applied Physics 108 (2010) 103711

[15] Yu.P. Gnatenko, R.V. Gamernyk, I.A. Farina, P.I. Babii, Physics of the Solid State 40 (1998) 1107

[16] J.A. Garcia, A. Remon, V. Munoz, R. Triboulet, Journal of Materials Research 15 (2000) 1612.

[17] Yu.P. Gnatenko, O.A. Shigil'chev, E. Rutkovski, G. Contreras-Puente, M. Cardenas-Garcia, Physics of the Solid State 40 (1998) 564.

[18] G.F. Vander Voort, Metallography Principles and Practice, ASM International, New York (1999) 1999; 445.

[19] Yu.P. Gnatenko, Yu.P. Piryatinski, R.V. Gamernyk, I.O. Faryna, P.M. Bukivskij, S.Yu. Paranchych, L.D. Paranchych, Proceedings of SPIE 5209 (2003) 156.

[20] Andrea Fasoli, Alan Colli, Faustino Martelli, Simone Pisana, Ping Heng Tan, Andrea C Ferrari, Nano Research 4 (2011) 343.

[21] R. Tenne, H. Mariette, C. Levy-Clement, R. Jager-Waldau, Physical Review B 36 (1987) 1204.

[22] Yoshihiro Kokubun, Hideo Watanabe, Masanobu Wada, Japanese Journal of Applied Physics 13 (1974) 1393.

[23] C.H. Henry, K. Nassau, J.W. Shiever, Physical Review B 4 (1971) 2453.

[24] R. Soundararajan, K.G. Lynn, S. Awadallah, C. Szeles, Su-Huai Wei, Journal of Electronic Materials 35 (2006) 1333.

[25] G. Mandel, F.F. Morehead, P.R. Wagner, Physical Review 136 (1964) A826.

[26] B. Segal, D.T.F. Marple, in: M. Aven, J.S. Prener (Eds.), Physics and Chemistry of II-YI Compounds, North-Holland, Amsterdam, 1967, p. 317.

[27] D. Berlincourt, H. Jaffe, L.R. Shiozawa, Physical Review 129 (1963) 1009.

[28] R.A. Burmeister Jr, D.A. Stevenson, Physica Status Solidi (b) 24 (1967) 683.

[29] L. Ion, S. Antone, M. Popescu, F. Scarlat, F. Sava, F. Ionescu, Journal of Optoelectronics and Advanced Materials 6 (2004) 113.

[30] S.M. Hus, M. Parlak, Journal of Physics D: Applied Physics 41 (2008) 035405.

[31] N.I. Tarbaev, Physics of the Solid State 40 (1998) 1672.

[32] G. Perna, V. Capozzi, European Physical Journal B 26 (2002) 401.

[33] A.V. Bazhenov, Yu.A. Osipjan, E.A. Shteinman, Soviet Physics-Solid State 13 (1972) 2683.

[34] R.E. Halstead, M. Aven, Physical Review Letters 14 (1965) 64. 